without active disease and $19 \%$ still experiencing active disease. $32 \%$ were still receiving GCs - $22 \%$ of them receiving $>5 \mathrm{mg} /$ day. There was negative impact on functional status with $14 \%$ reducing working hours, $13 \%$ restricted social life, $6 \%$ leaving employment, $6 \%$ registered as disabled and $2 \%$ leaving full time education. Conclusion: The start of maintenance treatment in AAV is variably defined but typically at 6 months after start of remission induction therapy. Achieving full remission and preventing relapse are still clinical problems and many patients require ongoing GC therapy to maintain remission. Infectious complications and adverse events are common and there is significant negative impact on patient functional status over time. Disclosure of Interests: Peter Rutherford Shareholder of: Vifor Pharma, Employee of: Vifor Pharma, Baxter Healthcare, Dieter Götte Shareholder of: Vifor Pharma, Employee of: Vifor Pharma

DOI: 10.1136/annrheumdis-2020-eular.844

\section{OP0030 \\ GRANULOMATOSIS WITH POLYANGIITIS SUSTAINED REMISSION OFF-THERAPY: DATA FROM THE FRENCH VASCULITIS STUDY GROUP REGISTRY}

X. Puéchal ${ }^{1}$, M. Iudici ${ }^{2}$, C. Pagnoux ${ }^{3}$, A. Karras ${ }^{4}$, P. Cohen ${ }^{1}$, F. Maurier ${ }^{5}$, T. Quéméneur ${ }^{6}$, F. Lifermann ${ }^{7}$, M. Hamidou ${ }^{8}$, L. Mouthon ${ }^{1}$, B. Terrier ${ }^{1}$, L. Guillevin ${ }^{1}$ ${ }^{1}$ Cochin Hospital, Paris, France, ${ }^{2}$ University Hospitals, Geneva, Switzerland; ${ }^{3}$ Mount Sinai Hospital, Toronto, Canada; ${ }^{4} \mathrm{HEGP}$, Paris, France; ${ }^{5} \mathrm{HP}$, Metz, France; ${ }^{6} \mathrm{CH}$, Valenciennes, France; ${ }^{7} \mathrm{CH}$, Dax, France; ${ }^{8} \mathrm{CHU}$, Nantes, France

Background: Data on granulomatosis with polyangiitis (GPA) sustained remission off-therapy (SROT) are limited and it is unknown whether disease characteristics or treatment regimen may affect it.

Objectives: This study aimed to assess SROT of GPA patients from the French Vasculitis Study Group registry, and identify factors associated with its occurrence and durability during follow-up.

Methods: GPA had to satisfy the 1990 ACR classification criteria and/or revised Chapel Hill Nomenclature for study inclusion. SROT was defined as remission (BVAS $=0$ ) without glucocorticoids (GC) or immunosuppressants (IS), the latter for $\geq 6$ months (ie 2 consecutive visits). SROT and its duration were extracted from the database. Data from patients with 3-, 5- and 10-year SROT were analyzed. Baseline characteristics of patients with 3-year GPA SROT were compared to those of registry GPA patients with available data at 3 years but not in SROT (controls), and 3-year SROT achieving 5-year SROT vs those who relapsed between $3 \& 5$ years. Patients with 3-year GPA SROT follow-up +7 years were analyzed according to maintained SROT or not.

Results: Among 795 database patients with new-onset GPA, 259 achieved at least 1 SROT at some time during their disease, after a median [IQR] of 36 [28-63] months post-diagnosis. The first SROT lasted a median of 14 [18-32] months. Among 202 of those patients who had follow-up, 73 (36\%) remained in SROT for a median follow-up of 34 [1445] months post-SROT. Among 434 (54\%) patients followed for $\geq 3$ years post-diagnosis, $82 \%$ had received GC and cyclophosphamide induction therapy. At 3 years post-diagnosis, $92(21 \%)$ patients in SROT were compared to $342(79 \%)$ controls who had relapsed or were still taking GC or IS. Patients achieving 3-year SROT vs controls, respectively, had more frequently received intravenous cyclophosphamide as induction therapy $(89 \%$ vs $77 \%, P=0.01$ ), with a higher median number of infusions ( 7.5 vs $6 ; P=0.05$ ); no other clinical or biological baseline difference was found. Among those 923 -year SROT patients, $74 \mathrm{had} \geq 2$ years of additional follow-up: 46 (62\%) attained 5-year SROT and 28 (38\%) had relapsed after a mean follow-up of 13 months. Baseline clinical and biological characteristics of patients achieving 5-year SROT did not differ from those of 3-year SROT patients who relapsed. Among those 923 -year SROT patients, $16 \mathrm{had} \geq 7$ additional years of follow-up: 6 (38\%) achieved 10-year SROT, ie 8\% of 75 GPA with available data at 10 years, and 10 (63\%) had relapsed a mean $35 \pm 28$ months after achieving 3-year SROT. Conclusion: Only $8 \%$ of GPA patients achieved 10 -year SROT after conventional induction and maintenance therapies. No baseline clinical or biological characteristics helped distinguish patients achieving or maintaining SROT and those who relapsed. However, patients achieving 3-year SROT had received more intensive induction therapy than those who relapsed or were still on GC or IS at 3 years. Disclosure of Interests: None declared DOI: 10.1136/annrheumdis-2020-eular.1827

\begin{tabular}{|l|l}
\hline OP0031 & AN INCREASE IN SERUM CALPROTECTIN LEVEL \\
IN ANCA-ASSOCIATED VASCULITIDES PATIENTS \\
DURING MAINTENANCE THERAPY IS ASSOCIATED \\
WITH MORE RELAPSE AND ACCELERATED RENAL \\
FUNCTION DECLINE
\end{tabular}

X. Romand ${ }^{1}$, A. Courtier ${ }^{2}$, M. V. C. Nguyen ${ }^{2}$, M. H. Paclet ${ }^{1}$, P. Gaudin ${ }^{1}$, L. Guillevin ${ }^{3}$, B. Terrier ${ }^{3}$, A. Baillet ${ }^{1} .{ }^{1}$ Univ. Grenoble Alpes, GREPI, Grenoble, France; ${ }^{2}$ Sinnovial, Grenoble, France; ${ }^{3}$ Cochin Hospital, Department of Internal Medicine, National Referral Center for Rare Systemic Autoimmune Diseases, Paris, France

Background: Calprotectin (S100A8/A9), a protein secreted by activated neutrophils and monocytes in inflammatory conditions, is upregulated in active ANCA-associated vasculitides. Serum calprotectin level variation during induction therapy is associated with disease relapse in PR3-ANCA-associated vasculitides (1). However, the place of this biomarker during maintenance therapy is unknown.
Objectives: To demonstrate whether variation in serum calprotectin level during maintenance therapy could be used as a biomarker predicting subsequent relapse in ANCA-associated vasculitides.

Methods: Patients with ANCA-associated vasculitides in complete remission (BVAS=0) after induction therapy with cyclophosphamide and included in the MAINRITSAN trial (2) were analyzed. Patients were randomized to receive rituximab or azathioprine as maintenance therapy. Relapse was defined as the re-occurrence or new onset of disease attributable to active vasculitis. Accelerated decline renal function (estimated Glomerular Filtration Rate (eGFR) assessed using the MDRD equation) was defined in concordance with NICE 2015 guideline (3) as "a decrease in eGFR of $25 \%$ or more and a change in GFR category or a sustained decrease in eGFR of $15 \mathrm{ml} / \mathrm{min} / 1.73 \mathrm{~m}^{2}$ over 12 months." Calprotectin was assessed in the serum at inclusion and 6 months by ELISA (IDK@ Calprotectin ELISA kit, Immunodiagnostik). We defined an increase in serum levels of calprotectin as a positive variation of calprotectin level at M6 compared to baseline. Results: Of all, 96 patients (female $45.8 \%$, mean age $55.3 \pm 13.5,69.8 \%$ PR3,$+ 62.5 \%$ ANCA positive at inclusion) had at least a calprotectin dosage (86 at baseline, 86 at M6 and 76 patients at this 2 time-point). Calprotectin level at baseline or 6 months was not significantly different between relapsing patients and those without relapse after 18 months of follow-up, whereas the calprotectin variation at M6 compared to baseline was higher in relapsing patients $(n=10)$ (mean (SD) $17991( \pm 28972) \mathrm{ng} / \mathrm{ml})$ than in patients not experiencing any relapse $(n=66)(9419( \pm 50002) n g / m L ; p=0.03)$. An increase in serum calprotectin level at 6 months was significantly associated with an increased risk of relapse in PR3-ANCA patients $(\mathrm{OR}=5.6(95 \% \mathrm{Cl}, 1.0-31.3 ; \mathrm{p}=0,049)$ but not in the whole study group $(\mathrm{OR}=3.3(95 \% \mathrm{Cl}, 0.8-14.1 ; \mathrm{p}=0.1)$, and identified patients with accelerated renal function decline (all cohort: $\mathrm{OR}=10.6(95 \% \mathrm{Cl}, 2.9-39.6$ $\mathrm{p}=0.002$; $\mathrm{PR} 3+$ patients: $\mathrm{OR}=5.909(95 \% \mathrm{Cl}, 2.9-39.6 ; \mathrm{p}=0.01))$, whereas calprotectin level did not correlate with glomerular filtration rate $(r=-0.07, p=0.35)$.

Conclusion: An increase in serum calprotectin during the first 6 months of maintenance therapy in ANCA-associated vasculitides is a useful biomarker predicting vasculitis relapse and accelerated renal function deterioration in the following 12 months.

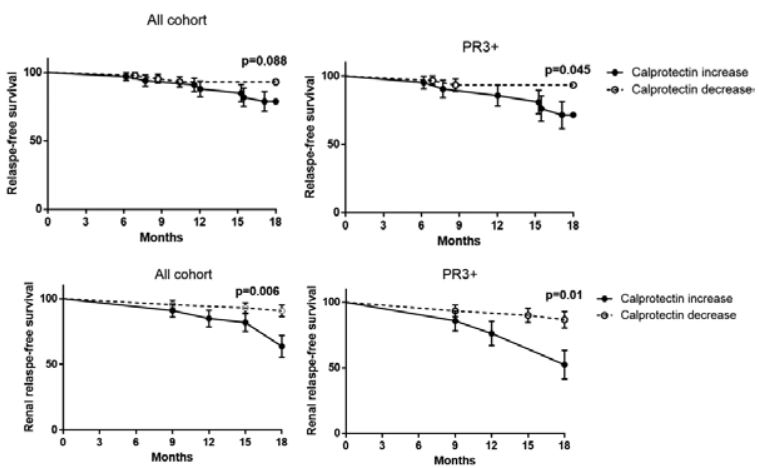

Increase calprotectin serum at 6 months identify relapser and accelerated decline renal function at 1 year. Kaplan-Meier survival curves of ANCA-associated vasculitis patients with an increase in serum calprotectin at 6 months (solid line) or not (dotted line) remaining total relapse-free or accelerated decline renal function-free. Gehan-Beslow-Wilcoxon test.

References:

[1] Pepper RJ et al. Arthritis Rheumatol. 2017;69(1):185-93.

[2] Guillevin L et al. N Engl J Med. 2014;371(19):1771-80.

[3] NIH. Chronic kidney disease 2014.

Acknowledgments: Supported by a grant from the Programme Hospitalier de Recherche Clinique, French Ministry of Health (2008-002846-51).

Disclosure of Interests: Xavier Romand Consultant of: Xavier ROMAND has received honorarium fees from Abbvie, Anais Courtier: None declared, Minh Vu Chuong Nguyen: None declared, Marie-Hélène Paclet: None declared, Philippe Gaudin Speakers bureau: Lilly, Loïc Guillevin: None declared, Benjamin Terrier: None declared, Athan Baillet Consultant of: Athan BAILLET has received honorarium fees from Abbvie for his participation as the coordinator of the systematic literature review DOI: 10.1136/annrheumdis-2020-eular.4083

\section{OP0032 \\ ERAP1-MEDIATED IMMUNOGENICITY AND IMMUNE- PHENOTYPES IN HLA-B51+ BEHÇET'S DISEASE POINT TO PATHOGENIC CD8 T CELL EFFECTOR RESPONSES}

A. F. Al-Obeidi ${ }^{1}$, A. Cavers ${ }^{1}$, Y. Ozguler ${ }^{2}$, O. Manches ${ }^{3}$, H. Zhong ${ }^{1}$, B. Yurttas ${ }^{2}$ B. Ueberheide ${ }^{1}$, G. Hatemi ${ }^{2}$, M. Kugler ${ }^{1}$, J. Nowatzky ${ }^{1} .{ }^{1} N Y U$ School of Medicine, New York, United States of America; ${ }^{2}$ Istanbul University Cerrahpassa Medical School, Istanbul, Turkey; ${ }^{3}$ Inserm, U 1209, Grenoble, France

Background: HLA-B51 is a definite risk factor for Behçet's disease (BD). A coding variant of ERAP1, Hap10 - with low peptide-trimming activity - vastly potentiates this risk, but is mechanistically unclear ${ }^{1,2)}$ 